\title{
Rompre avec les politiques néolibérales dans le domaine des relations industrielles
}

L'expérience de la Grande-Bretagne, de l'Australie et de la NouvelleZélande

Drawing Back from Neo-liberal Industrial Relations Change: the Experience of

Britain, Australia and New Zealand

Peter Waring, John Lewer et John Burgess

\section{(QpenEdition \\ Journals}

Édition électronique

URL : http://journals.openedition.org/travailemploi/4810

DOI : 10.4000/travailemploi.4810

ISSN : 1775-416X

Éditeur

DARES - Ministère du Travail

Édition imprimée

Date de publication : 15 décembre 2010

Pagination : 5-15

ISSN : 0224-4365

Référence électronique

Peter Waring, John Lewer et John Burgess, « Rompre avec les politiques néolibérales dans le domaine des relations industrielles », Travail et Emploi [En ligne], 124 | octobre-décembre 2010, mis en ligne le 15 décembre 2012, consulté le 23 avril 2019. URL : http://journals.openedition.org/travailemploi/4810 ; DOI : 10.4000/travailemploi.4810 


\title{
Rompre avec les politiques néolibérales dans le domaine des relations industrielles. L'expérience de la Grande-Bretagne, de l'Australie et de la Nouvelle-Zélande
}

\author{
Peter Waring, John Lewer, John Burgess (*)
}

\begin{abstract}
L'élection entre le milieu des années 1990 et la fin des années 2000 de partis travaillistes en Australie, Nouvelle-Zélande et Grande-Bretagne, après des vagues de réformes libérales qui ont marginalisé les syndicats et remodelé les structures de la négociation sociale en faveur du capital, a apporté la promesse d'une intervention accrue de l'État et d'une plus grande équité dans les relations industrielles. En Australie, le gouvernement conservateur de John Howard, en place depuis onze ans, subit en novembre 2007 une défaite cuisante. La campagne avait été marquée par un débat intense sur la réforme des relations professionnelles, les Work Choices, intervenue en 2005. Les Work Choices ont produit les mêmes effets en Grande-Bretagne et en Nouvelle-Zélande, où les électeurs avaient en 1996 et 1999 largement voté contre la législation néolibérale en matière de relations industrielles. Ce rééquilibrage voulu entre valeurs d'équité et valeurs d'efficacité n'a cependant pas empêché ces pays de conserver une part importante de l'héritage néolibéral. Ceci met en lumière les difficultés qu'il y a à revenir en arrière sur des dispositions législatives et à reconstruire des institutions qui ont été démantelées, et dans le même temps, pour ces nouveaux gouvernements, à faire montre de pratiques de gestion économique fiables.
\end{abstract}

La Grande-Bretagne, l'Australie et la NouvelleZélande, pays d'économie capitaliste, ont aussi connu des histoires proches sur le plan constitutionnel et politique (avec un même principe de pluralisme) et partagent les mêmes traditions du Common Law. Castles et Pierson (1996, p. 234) notent qu'ils « tendent à avoir des gouvernements émanant d'un seul parti, dont émane le chef de l'exécutif $\gg$. Au-delà de ces similitudes, chacun des pays a développé sa propre approche des relations industrielles et ses propres mécanismes institutionnels en la matière ; approches qui ont depuis connu des changements, voire de fortes transformations, dans un cadre néolibéral. Des programmes de réformes néolibérales ont été mis en ouvre en Grande-Bretagne par le gouvernement Thatcher (1979-1996), en Nouvelle-Zélande par le National Party (1990-1999) et en Australie par le gouvernement Howard (1996-2007). Certains éléments de ces réformes ont toutefois préexisté à ces gouvernements : le gouvernement Hawke-Keating en Australie (1983-1996), par exemple, a adopté des politiques néolibérales telles que la dérégulation du secteur financier et la privatisation. Mais pour l'essentiel, les changements radicaux des systèmes

(*) Université de Newcastle, Australie ; peter.waring@newcastle. edu.au ; john.lewer@newcastel.edu.au ; john.burgess@ newcastle. edu.au de relations industrielles dans chacun de ces pays proviennent des gouvernements conservateurs de Margaret Thatcher, du National Party et de John Howard. Nous verrons en détail comment tous les trois ont été battus (1) lors d'élections législatives et remplacés par des partis travaillistes sociaux-démocrates ayant pour mandat d'amender les politiques de relations industrielles néolibérales qu'ils avaient mises en place.

Après avoir rappelé les caractéristiques-clés du néolibéralisme, nous examinerons dans chaque pays les changements apportés pour tenter de réformer les systèmes néolibéraux de relations industrielles, et proposerons une comparaison entre eux ; puis nous listerons une série de facteurs ayant pu affecter le retour à un régime de "re-régulation ». En substance, cet article s'interroge, à partir de l'expérience de la Grande-Bretagne, de l'Australie et de la Nouvelle-Zélande, sur la possibilité de revenir à des relations industrielles plus régulées et plus collectives après une transformation aussi importante du contexte économique et social et des institutions. Nous employons le terme de « re-régulation » dans un sens générique, pour décrire le « retour en arrière » opéré lors de la phase postnéolibérale qui

(1) Il convient de noter que la majorité a de nouveau changé en 2010 au Royaume-Uni, le conservateur David Cameron ayant remplacé comme premier ministre le travailliste Gordon Brown. 
a suivi la défaite des gouvernements conservateurs ; nous n'entendons pas par là que le néolibéralisme se caractérise toujours par la mise en œuvre d'une régulation moins importante : cela n'a pas été le cas par exemple des Work Choices australiens, où des lois nombreuses et complexes ont été adoptées, qui encadrent notamment le fonctionnement du marché du travail.

\section{Le néolibéralisme}

Le néolibéralisme est généralement présenté comme un système fondé sur l'allocation des ressources par le marché et sur l'individualisme, avec peu d'interventions du gouvernement, peu d'impôts et des dispositions strictes sur les transferts sociaux (GEORGE, 1999). Les politiques gouvernementales néolibérales reposent sur la domination du marché en tant que meilleur mécanisme d'allocation des ressources et vecteur d'efficacité. Ainsi, le secteur public doit s'aligner plus étroitement sur les impératifs économiques, avec par exemple, la transformation des entreprises publiques en sociétés anonymes ; des politiques de libre-échange sont menées et les contraintes sur la mobilité des capitaux et des investissements sont réduites (GEORGE, 1999). Comme l'indique LARner (2000, p. 6), « le néolibéralisme est à la fois un discours politique sur la nature des règles et une série de pratiques facilitant la gouvernance des individus à distance ». Dans l'analyse détaillée qu'il en fait, Mudge (2008) met en évidence trois dimensions interconnectées du néolibéralisme : la dimension intellectuelle, la dimension bureaucratique et la dimension politique. La dimension intellectuelle est anglo-américaine et liée au marché en tant qu'arbitre de la réussite ; la dimension bureaucratique englobe les politiques des États favorables aux marchés, comme la dérégulation, la privatisation et la libéralisation ; la dimension politique met en avant les politiques axées sur le marché et la limitation des compétences de l'État. Mudge (2008) soutient que ces trois aspects du néolibéralisme ne peuvent être séparés et que le néolibéralisme est une idéologie globale qui ne se limite pas aux partis politiques conservateurs.

Pour GedDEs (2005), les différences dans la façon dont le néolibéralisme s'est déployé dans les différents pays sont particulièrement visibles dans les régimes de transferts sociaux. Ainsi, alors que les États-Unis, le Royaume-Uni, l'Australie et la Nouvelle-Zélande partagent des valeurs néolibérales de base, leur approche présente des différences quant à l'étendue de ces transferts, à la définition des programmes prioritaires en matière de lutte contre la pauvreté et au lien entre citoyenneté et responsabilité. Ces différences d'approche peuvent également s'analyser à partir de certains textes de base de la littérature sur les variétés du capitalisme
(Hall, Soskice, 2001), qui distinguent les « économies de marché libérales » présentes aux États-Unis, en Grande-Bretagne et en Australie, et les « économies de marché coordonnées » existant en Europe continentale et au Japon, dont le financement par emprunt à long terme est plus important et le droit du travail plus protecteur (WAILES, KITAY, LANSBURY, 2008).

Tout en admettant que le terme « néolibéralisme » a des significations très nombreuses et que sa mise en œuvre a considérablement varié selon les pays, nous pensons néanmoins qu'il reflète bien les changements opérés par les gouvernements conservateurs britannique, australien et néo-zélandais, notamment par l'accent mis sur l'individualisme, la privatisation, la restructuration de l'État-providence et la place du marché dans l'économie. Pour ce qui concerne les systèmes de relations industrielles, même si les programmes néolibéraux différaient, chacun de ces pays a connu une tentative concertée de transformation fondamentale. La nécessité de réformer ces systèmes a été le fil rouge commun des régimes conservateurs, qui s'est effectuée par le soutien à des prérogatives patronales nettement accrues, la limitation du champ de la négociation collective et l'affaiblissement de la place des syndicats dans le marché du travail grâce à l'intensification de la négociation individuelle, sans intervention syndicale.

Cet article fournit un résumé analytique de décennies de changements survenus dans ces trois pays, sous des gouvernements conservateurs et travaillistes, et met l'accent sur les évolutions les plus marquantes. Il ne cherche pas à élaborer une explication théorique globale de ce qui s'est passé, mais apporte un ensemble d'explications dont certaines ont encore un caractère hypothétique.

\section{Le contexte institutionnel : partis de centre-gauche, néolibéralisme et syndicats}

Certaines différences institutionnelles fondamentales entre ces trois pays méritent notre attention. Tout d'abord, la Nouvelle-Zélande et le RoyaumeUni ont des systèmes nationaux simples, tandis que celui de l'Australie consiste en une fédération de gouvernements des États et du Commonwealth. Le Royaume-Uni et l'Australie ont tous deux des systèmes parlementaires à deux chambres, dont la chambre haute a des compétences de contrôle. La Nouvelle-Zélande est unicamérale. Le RoyaumeUni est également membre et signataire des traités de l'Union européenne. La Nouvelle-Zélande et l'Australie ont des obligations internationales découlant, par exemple, de leur adhésion à l'Organisation des Nations unies, mais qui n'opèrent généralement pas avec la même force que l'Union 
européenne sur le Royaume-Uni. Ensuite, il existe des différences tout à fait considérables entre les économies de ces pays. L'économie australienne, par exemple, est fortement liée à l'exportation de produits de base ; la Nouvelle-Zélande a de forts liens commerciaux avec l'Asie, et le commerce britannique est orienté vers l'Union européenne. Enfin, en termes de relations industrielles, l'Australie et la Nouvelle-Zélande ont eu un système de régulation obligatoire passant par l'intermédiaire de tribunaux de conciliation et d'arbitrage. À l'inverse, le système britannique a évolué vers une régulation collective au niveau des branches.

Ces trois pays ont en outre un duopole politique où les partis conservateurs sont opposés aux partis travaillistes de centre-gauche affiliés ou associés à des syndicats. Au cours des vingt dernières années, les partis travaillistes ont été longtemps au pouvoir - du milieu des années 1980 au milieu des années 1990 en Australie, depuis 1996 au Royaume-Uni, et de 1999 à 2008 en Nouvelle-Zélande -, périodes pendant lesquelles ces trois pays ont étonnamment appliqué des programmes néolibéraux sur le marché des produits et le marché financier, ainsi que sur l'encadrement des politiques monétaires, fiscales et sociales (Mudge, 2008).

\section{La Grande-Bretagne après le parti conservateur}

Le gouvernement conservateur de Margaret Thatcher, à travers une succession de lois, a transformé le système de relations industrielles en Grande-Bretagne, en exerçant un contrôle beaucoup plus strict des syndicats, en limitant leur accès aux lieux de travail, en réduisant leur possibilité d'engager des grèves et en modifiant leur force de proposition en matière de négociation collective (Shackleton, 1998 ; Deakin, 1992 ; Dickens, Hall, 1995). Une nouvelle loi sur relations industrielles était promulguée tous les deux ans en moyenne. La loi sur l'emploi (Employment Act) de 1980 supprimait les procédures de reconnaissance syndicale obligatoire, restreignait l'application du closed shop (monopole d'embauche de personnes affiliées à un syndicat) et prévoyait, quand un syndicat proposait de lancer un mouvement de grève, de financer sur crédits publics le vote à bulletin secret de ses adhérents. Elle a été suivie de l'Employment Act de 1982 qui réduisait encore l'application des closed shops et les protections légales accordées aux syndicats. La loi sur les syndicats (Trade Unions Act) de 1984 a étendu le vote à bulletin secret aux élections syndicales, aux grèves et aux dons offerts par des partis politiques. L'Employment Act de 1990 a autorisé le licenciement en cas de grève non autorisée, a rendu illégal le boycottage secondaire et a étendu la responsabilité des syndicats en cas de grève non autorisée. La loi de 1993 sur la réforme syndicale et les droits en matière d'emploi (Trade Union Reform and Employment Rights Act) a appliqué d'autres restrictions aux votes sur les grèves et a aboli les conseils des salaires. SHACKLETON (1998, p. 588) conclut que ce programme continu de changements législatifs a entraîné l'érosion progressive du pouvoir et de la position des syndicats, associée à la disparition du soutien à la négociation collective, qui a vu « les droits individuels [...] renforcés à la place des droits collectifs ».

Le pouvoir syndical s'est également heurté au gouvernement à l'occasion de la privatisation croissante des services publics et l'extension du recours à la sous-traitance (DEAKIN, 1992) ; l'abolition des commissions de formation professionnelle, qui comprenaient des représentants syndicaux, s'est accompagnée de leur remplacement par des conseils de formation et d'entreprise dominés par les employeurs (SHACKLETON, 1998) ; et il faut bien sûr citer l'action vigoureuse de l'État en réponse à la grève des mineurs de 1984-1985. À cet égard en 1997, le taux de syndicalisation était passé de $53 \%$ à $30 \%$ (SHACKLETON, 1998).

Le gouvernement travailliste de Tony Blair a été élu en 1997, en grande partie grâce aux efforts fructueux mis en œuvre pour attirer les électeurs centristes. Cette stratégie a nécessité la reconstitution de l'image du parti travailliste, qui s'est s'éloigné du mouvement travailliste britannique et a promis des politiques qui apporteraient des changements aux réformes du gouvernement Thatcher plus qu'ils ne s'y substitueraient ( $c f$. SMITH, MORTON, 2001). Toutefois, la promulgation de la loi de 1999 sur les relations industrielles (Employment Relations Act ou ERA), faisant suite à la publication du Livre blanc consacré à l'équité au travail (Fairness at Work), représentait en quelque sorte un rééquilibrage entre les intérêts du travail et du capital, rééquilibrage qui s'inscrivait dans la philosophie du «partenariat social » et dans le tournant de la « Troisième voie » (Third Way) vers la re-régulation du marché du travail britannique ( $c f$. par exemple, Brown, 2000). Le gouvernement Blair a également établi une Commission des bas salaires (Low Pay Commission) en juin 2000, chargée d'enquêter sur les questions liées à la mise en place d'un salaire minimum national.

L'influence du courant de pensée de la Troisième voie sera particulièrement visible dans les mesures précises de l'ERA et les valeurs qu'il porte. Par exemple, les changements législatifs incorporés à cette loi étaient principalement orientés vers l'extension des droits individuels plutôt que vers des mesures visant à faciliter l'action collective sur les lieux de travail. Cette nouvelle loi n'a pas éliminé les contraintes sur les grèves décidées par les précédents gouvernements conservateurs, la plupart des restrictions législatives du gouvernement Thatcher 
concernant les grèves restant en vigueur (BROwN, 2000, p. 301). SMith et MorTon (2001, p. 131) affirment qu'avec cette loi, " la liberté des travailleurs individuels à participer à une action industrielle reste fragile », voire qu'elle " ne respecte pas les normes internationales ». De la même manière, UNDY (1999, p. 330) soutient que le maintien des restrictions en matière de grève montre que le gouvernement Blair « considère comme approprié l'équilibre du pouvoir de négociation en faveur du patronat ». Pourtant, cette loi introduisait également un système de reconnaissance syndicale obligatoire, en dépit de l'opposition des employeurs, cohérente avec l'idée du New Labour selon laquelle les salariés devraient avoir le droit de choisir s'ils souhaitent être représentés ou non par un syndicat. En outre, ce mécanisme était également cohérent avec l'objectif du gouvernement de "promouvoir une nouvelle culture de partenariat sur les lieux de travail » (WoN, GoDDARD, 1999, p. 204).

Bien que le courant de pensée de la Troisième voie ait eu une grande influence sur la plate-forme du gouvernement Blair pour le renouveau socialdémocrate ( $c f$. GidDENs, 1998), sa mise en œuvre a suscité un débat considérable. UNDY (1999) fait l'hypothèse que le fait que la Troisième voie n'ait pas mis l'accent sur les relations industrielles est un signe qu'elles ne sont plus une question essentielle. La mondialisation et la désintégration régulière de toutes les formes d'action collective, selon UNDY, sont le signe que les partisans de la Troisième voie ont relégué les relations industrielles au rang de questions dénuées de pertinence. Toutefois, il semble que les implications indirectes de la philosophie de la Troisième voie soient perceptibles dans l'approche du gouvernement Blair concernant les relations industrielles. Pour Undy, la réponse du New Labour aux dilemmes de Giddens (mondialisation et individualisme) se trouve dans les accords sur les relations industrielles, d'abord avec l'adoption du Chapitre social de l'Union européenne en 1997, ensuite avec l'extension des droits individuels au travail.

McKAY (2001, p. 291) soutient que la signature du Chapitre social par le New Labour a marqué son acceptation de la «dimension sociale de l'intégration européenne » et a poussé le gouvernement Blair à introduire une législation cohérente avec les directives européennes sur des questions telles que l'organisation du temps de travail, les congés parentaux et, sujet peut-être plus controversé, les comités d'entreprise. La directive sur les comités d'entreprise européens est entrée en vigueur le 15 janvier 2000 au Royaume-Uni ; elle prévoit que les entreprises employant 1000 salariés ou plus (environ 300 sociétés établies au Royaume-Uni) instituent des comités d'entreprise composés de représentants des salariés et de la direction (McKAY, 2001). La mise en œuvre de cette directive semble également tout à fait cohérente avec l'enthousiasme du gouvernement Blair et l'objectif de l'ERA consistant à promouvoir le partenariat sur les lieux de travail et à remplacer les relations professionnelles de confrontation par des notions de coopération et de gains mutuels (Guest, Peccei, 2001).

Il ne fait aucun doute que le retournement du New Labour a été encouragé par la réglementation sociale européenne. Mais, ici aussi, on a pu observer une approche plutôt minimaliste face à la mise en œuvre de cette réglementation. La question est de savoir si ces nouveaux droits sont le fruit de la démarche de la Troisième voie ou simplement le résultat des directives de l'Union européenne. BROWN (2000) soutient qu'ils reflètent la pression des directives européennes tandis que McKaY (2001, p. 291) affirme que, même si le New Labour a signé le Chapitre social en 1997, il a « appliqué les propositions de l'Union européenne de manière minimaliste, sans jamais aller plus loin que ce qu'exigeait la législation européenne ».

Globalement, après la défaite du gouvernement conservateur, le gouvernement travailliste britannique a conservé une forte relation au néolibéralisme et à la centralité des marchés dans l'élaboration des politiques publiques. Des éléments-clés du répertoire néolibéral d'action publique ont persisté : la privatisation des entreprises publiques, la séparation entre l'autorité de régulation et le pouvoir exécutif (par exemple, la banque centrale indépendante), la dépolitisation de la régulation économique (par exemple, une autorité indépendante en matière de concurrence) et l'ouverture des marchés à plusieurs fournisseurs de services (par exemple, les partenariats public-privé) (MudGE, 2008). Soustraire le marché du travail et le régime de relations industrielles d'un tel agenda politique n'est pas possible: aussi la Troisième voie a-t-elle représenté une forme nuancée de néolibéralisme, bien qu'inscrite dans le cadre de la réglementation sociale européenne. En ce qui concerne la reconnaissance syndicale et le droit de déclencher une grève, le bilan est contrasté. Les relations entre le parti travailliste et le monde du travail ont été au mieux tièdes, et il n'y a eu aucun retour aux droits et privilèges dont les syndicats bénéficiaient avant la période Thatcher. Ainsi, la Commission des bas salaires, qui a initié l'augmentation des salaires pour les travailleurs faiblement rémunérés, la réduction des inégalités salariales et la diminution de l'écart de salaire entre les sexes (Metcalfe, 2008), représente peut-être la contribution la plus importante du New Labour au programme de justice sociale, avec l'adoption d'objectifs traditionnels d'équité et l'apport d'éléments jusque-là absents du cadre des relations industrielles du Royaume-Uni.

En mai 2010, le parti travailliste britannique a dû laisser la place à une coalition formée par le parti conservateur et le parti libéral-démocrate. Les observateurs ont pronostiqué de possibles tensions 
au sein de la coalition, dans la mesure où les libéraux-démocrates, favorables à un certain interventionnisme dans le domaine des politiques de relations industrielles, devront s'accommoder de la préférence des conservateurs pour moins de réglementation (Prosser, 2010). Ceci dit, le Congrès des syndicats (Trade Union Congress) a répondu par une campagne de mobilisation nationale de résistance (Trade UnION Congress, 2011) à la réduction des dépenses publiques annoncée par le nouveau gouvernement en réponse à la récession économique mondiale.

\section{L'Australie après les Work Choices}

Si changer la direction et l'agencement des structures de négociation a été une priorité essentielle des deux bords politiques australiens depuis le milieu des années 1980, le débat s'est intensifié avec la promulgation en 1996, par le gouvernement de coalition parti national/parti libéral de John Howard, de la loi sur les relations de travail (Workplace Relations Act ou WRA) et, par la suite, de la législation des Work Choices. Le WRA a limité les pouvoirs de la Commission australienne des relations industrielles, a diminué les pouvoirs de représentation des syndicats et a réduit l'étendue et l'application de conventions collectives aux salariés (comme les décisions des conseils de prud'hommes concernant les règles de travail et de rémunération). L'innovation peut-être la plus controversée de la loi de 1996 a été le tournant vers l'individualisation de la relation de travail, avec l'introduction législative de contrats individuels connus sous le nom d'Australian Workplace Agreements (AWA). Cette mesure permettait aux employeurs de proposer des contrats individuels à leurs employés qui, s'ils les acceptaient, étaient inscrits auprès de l'Office of the Employment Advocate (OEA), un nouvel organisme créé dans le cadre du WRA de 1996 ( $c f$. PeETz, 2006). Les AWA étaient des contrats privés et secrets qui incluaient la possibilité de pénalités légales contre une partie qui en aurait divulgué le contenu à des tiers. Les AWA permettaient aux employeurs, dans les limites de liberté d'association établies dans le WRA, de réduire l'implication syndicale, de supprimer certaines conditions d'embauche et d'étendre les prérogatives patronales. Malgré la volonté du gouvernement de promouvoir les AWA, le nombre de contrats signés est resté plutôt bas, bien qu'ils aient trouvé un certain écho dans le secteur de l'exploitation minière du fer et du charbon.

En octobre 2004, le gouvernement de coalition d'Howard a remporté son quatrième mandat successif, s'assurant ainsi la majorité aux deux chambres du Parlement, une situation rare en Australie. Cela a permis à la coalition des partis national/libéral de promulguer une nouvelle législation sans avoir à affronter l'opposition d'un Sénat hostile, en donnant au gouvernement une occasion historique de mettre en place une législation plus radicale dans le domaine des relations industrielles. Cette opportunité s'est concrétisée en 2006 par l'entrée en vigueur du Workplace Relations Amendment (Work Choices) Act de 2005. Plutôt que d'adopter une démarche de forte dérégulation avec un retour au droit commun des contrats, les Work Choices ont introduit un style de régulation par «commandement et contrôle » dans lequel l'Etat cherchait à contrôler les acteurs des relations industrielles et, par là, le résultat des négociations sociales. Globalement, les Work Choices visaient à instituer un changement profond défiant les syndicats de façon tout à fait inédite. Cette législation cherchait, entre autres, à développer les contrats individuels tout en affaiblissant les négociations et conventions collectives; elle a mis fin à l'arbitrage obligatoire, a supprimé la protection des salariés en cas de licenciement abusif dans les entreprises de 100 salariés ou moins, et introduit de nouvelles normes restrictives en matière de protection minimale (PeETz, 2006).

Les Work Choices ont rapidement mobilisé le mouvement syndical, qui a répondu par la campagne Your Rights@Work (vos droits au travail). Cette campagne, qui a coûté plusieurs millions de dollars, a été financée en grande partie par les cotisations des adhérents ; elle a mis en œuvre des moyens d'action importants (rassemblements de masse, tracts, panneaux d'affichage, autocollants, publicité à la radio, à la télévision et sur internet), et a tenté d'influencer la campagne pour les élections fédérales de 2007. Les publicités télévisées financées par les syndicats mettaient généralement en scène des salariés victimes d'un licenciement abusif et qui se retrouvaient sans aucune ressource, ou dont la vie familiale avait été perturbée par les demandes injustes de leur employeur quant à l'organisation du temps de travail. Ces publicités se sont généralement révélées efficaces ; BRETT (2007, p. 74) observe que « les sondages d'opinion indiquaient régulièrement que seuls $20 \%$ des électeurs étaient favorables aux Work Choices ». Pendant toute la campagne électorale, la législation des Work Choices a été un sujet à la fois discordant et déterminant, qui a clairement différencié les deux principaux partis (MACCALLUM, 2007). Lors de l'élection du 25 novembre 2007, un sondage sorti des urnes indiquait que, pour $78 \%$ des votants, les Work Choices étaient l'une des trois principales priorités, $45 \%$ déclarant qu'il « s'agissait de leur première priorité »(WEST, 2007). Pour les commentateurs politiques, les relations industrielles, et en particulier les réformes des Work Choices, ont joué un rôle central dans la défaite électorale du gouvernement Howard face à l'opposition menée par Kevin Rudd ( $c f$. MACCALLUM, 2007 ; West, 2007 ; BRETT, 2007).

En décembre, l'Australian Labor Party (ALP) de Kevin Rudd s'installe au gouvernement ; 
en février de l'année suivante, il a déjà pris les premières mesures législatives visant à annuler les Work Choices, avec le projet d'amendement de la loi sur les relations de travail "Transition vers un avenir équitable » de 2008 (Workplace Relations Amendment (Transition to Forward with Fairness) Bill 2008). Ce projet était une mesure provisoire applicable jusqu'au remplacement plus complet du Workplace Relations Act. Cette première action a supprimé les contrats individuels et a étendu les conditions relatives à la protection minimale.

La législation de transition du gouvernement Rudd et ses promesses de réformes plus profondes en 2010, telles qu'elles figurent dans la « Politique pour un futur équitable (Forward with Fairness) de l'ALP, laissent entendre que si les mesures de protection de l'équité figurent en bonne place dans son programme, il n'y aura pas de retour à l'arbitrage ou aux privilèges accordés aux syndicats au $\mathrm{XX}^{\mathrm{e}}$ siècle. Elle prévoit plutôt de développer une plus forte et plus ample protection minimale, ainsi que des normes instituant un salaire national minimum, et une plus grande attention à la négociation collective et aux droits sur les lieux de travail qu'elle implique. Le gouvernement reste engagé dans un programme de «modernisation » qui englobera les droits statutaires. Cela traduit en partie la baisse d'influence des syndicats sur le lieu de travail, la densité syndicale comme au Royaume-Uni ayant baissé aux cours des trois dernières décennies. Il n'y aura pas de retour à une Commission australienne des relations sociales occupant une position centrale ni au système de convention collective. Bien que les relations professionnelles aient été un thème fondamental de la campagne électorale et aient largement contribué au succès de l'ALP, les mesures prises pendant la transition restent modérées et de nombreux éléments-clés du programme conservateur sur les relations professionnelles sont restés en vigueur.

Il est intéressant également d'examiner dans quelle mesure l'ALP a suivi ses homologues britanniques dans l'adoption d'une « troisième voie » politique de relations industrielles. L'une des différences entre le New Labour et l'ALP réside clairement dans leurs positions par rapport à la négociation collective. Si sa promotion constitue un élément-clé de la politique Forward with Fairness de l'ALP, SMith et MorTon (2001) font remarquer que l'Employment Relations Act britannique ne l'encourage pas. Pour eux (ibid., p. 125), cette loi a réduit la portée de la négociation collective aux « relations (économiques) sur le marché, à l'exclusion des relations managériales ». Les différences entre les propositions de réformes du gouvernement travailliste de Rudd et celles du New Labour trouvent peut-être leur origine dans la conviction ferme et presque universelle que la défaite du gouvernement Howard est due à la législation radicale des Work
Choices et à la campagne syndicale efficace qui a été menée contre celle-ci.

\section{La Nouvelle-Zélande après la loi sur les contrats de travail (Employment Contracts Act)}

Le parti travailliste néo-zélandais (en coalition avec le parti Alliance) est arrivé au pouvoir en novembre 1999 sous la direction d'Helen Clark, formant ainsi le premier gouvernement travailliste depuis 1990. Il faisait suite à plusieurs années de réformes néolibérales qui avaient eu pour effet de démanteler quasi totalement les principales institutions qui régissaient autrefois les relations industrielles en Nouvelle-Zélande. De tous les pays de l'OCDE, la Nouvelle-Zélande est celui qui a appliqué les « réformes » néolibérales les plus radicales, en supprimant notamment les tribunaux de conciliation et d'arbitrage actifs depuis la fin du XIX ${ }^{\mathrm{e}}$ siècle. Il faut noter que ce processus avait déjà été initié en 1984 par un gouvernement travailliste. En effet, rejetant toute forme de coopération corporatiste avec l'aile syndicale du mouvement travailliste, ce gouvernement avait entrepris partiellement de déréguler le marché du travail en promulguant en 1987 la loi sur les relations de travail (Labour Relations $A c t$ ). Il avait néanmoins fait le choix de conserver un certain nombre de mécanismes de sécurité syndicale, dont le droit exclusif de négociation et l'obligation de cotiser à un syndicat. Le principal changement introduit alors était la suppression de l'arbitrage obligatoire : une mesure qui devait permettre, selon le gouvernement, de créer des structures de négociation décentralisées. Néanmoins, sous l'effet notamment de l'action syndicale, en choisissant par exemple de privilégier les accords collectifs de branche plutôt que les accords d'entreprise pour régir les conditions de travail, cette dérégulation a finalement eu des résultats limités. Les groupes de représentation des intérêts du capital comme le New Zealand Business Roundtable ont alors manifesté leur mécontentement vis-à-vis d'un gouvernement qui ne parvenait pas à produire un changement suffisant en matière de dérégulation, par rapport aux résultats obtenus sur le marché financier ou le marché des produits. Selon eux, le marché du travail continuait de souffrir d'un manque de souplesse et de certaines rigidités préjudiciables à l'emploi et ils estimaient qu'une réforme radicale s'imposait (Bray, WALSh, 1998).

Le militantisme et le mécontentement du patronat face à la ténacité des syndicats ont poussé le parti national conservateur, dès son succès électoral d'octobre 1990, à concentrer ses efforts sur une politique de dérégulation radicale. C'est dans ce cadre qu'a été promulguée en 1991 la loi sur les contrats de travail (Employment Contracts Act, ci-après « loi ECA ») : 
une loi qui est venue défaire un siècle de droits syndicaux et bouleverser le contexte législatif en substituant à l'approche collective (accords collectifs standardisés négociés par les syndicats) une orientation plus individualiste (contrats individuels négociés indépendamment des syndicats). L'ECA allait à l'encontre de l'ancienne loi sur les relations de travail de 1987 (Labour Relations Act) qui insistait sur l'importance de définir des procédures pour « conduire de façon ordonnée les relations entre employeurs et salariés » et soulignait la responsabilité des parties : elle visait à promouvoir un marché du travail efficace, la liberté d'association et le choix, par les salariés du ou des individus (s'ils choisissaient seulement de se faire représenter) autorisé(s) à les représenter. L'ECA a cassé la prérogative de représentation exclusive donnée aux syndicats en reconnaissant aux travailleurs la possibilité de choisir unilatéralement leurs agents de négociation. Elle a aboli l'obligation d'adhérer à un syndicat et a généré une vague de négociations individualisées, largement encouragée par de nouvelles dispositions. Le changement néolibéral a été beaucoup plus profond qu'au Royaume-Uni ou en Australie. Cela s'explique par l'impact, au Royaume-Uni, de la Charte des droits fondamentaux de l'Union européenne mais aussi, par opposition à l'Australie, à l'existence d'un système parlementaire à chambre unique en Nouvelle-Zélande.

L'ECA a considérablement marginalisé le système des relations industrielles de NouvelleZélande par rapport au courant dominant au sein des pays de l'OCDE. Dès 1997, l'ancien système propre à la Nouvelle-Zélande et autrefois stable était totalement révolu, les conventions signées avec un seul employeur et autres contrats individuels ayant largement supplanté les conventions collectives multi-entreprises et les accords collectifs de branche. Les chiffres cités par HonEYBOnE (1997), par exemple, indiquent que la part des travailleurs couverts par des conventions collectives et accords de branche négociés collectivement par les syndicats est passé de $59 \%$ en mai 1991 à seulement $6 \%$ en août 1993. Le nombre de syndicats a chuté de 227 à 83 (en partie sous l'effet d'opérations de fusions) et le taux de syndicalisation est passé à $20 \%$ des travailleurs, contre plus de $44 \%$ au milieu des années 1980 (Honeybone, 1997 ; Bray, WALsh, 1998). Le nombre d'interruptions de travail est passé de 193 en 1986 - avec 366000 jours ouvrés perdus - à 42 en 1997 - avec 24000 jours ouvrés perdus - (BRADFORD, 1999, p. 160). Selon l'analyse de BoxAll (2001, p. 27), l'ECA a intensifié les disparités liées aux compétences sur le marché du travail. Les individus les plus compétents étant « récompensés » et assurés d'une « position confortable » tandis que les moins compétents ou simplement les moins bien organisés éprouvaient «plus de difficultés à faire correctement entendre leur voix et, de façon générale, à se prévaloir de leurs droits sociaux ».
Dans l'ensemble, Fryer et HAYNES (2000, p. 122) concluent que «l'ECA a balayé les vestiges de l'ancien système pour le remplacer par un système décentralisé, fondé sur une base purement contractuelle et sur l'individualisation du contrat de travail [...], l'impact de l'ECA a été dramatique ».

Après les élections de 1999, le gouvernement de coalition formé par les travaillistes et le parti Alliance a abrogé l'ECA pour promulguer un nouveau texte, l'Employment Relations Act (ci-après « loi ERA ») en 2000, dès la première année de sa mandature. Cette nouvelle loi fixait un certain nombre d'objectifs-clés, parmi lesquels : «Établir des relations de travail productives en encourageant la confiance mutuelle dans tous les aspects de l'environnement de travail [...] ; reconnaître le fait que les relations de travail sont basées sur la bonne foi de chacun ; reconnaître l'existence d'une inégalité inhérente à la relation de travail en termes de pouvoir de négociation et tâcher de la compenser en encourageant les négociations collectives ».

Consécutivement à l'ERA, trois constats peuvent être dressés dans l'analyse du système de relations sociales néo-zélandais. Premièrement, le déclin spectaculaire du taux de syndicalisation suite à l'adoption de la loi ECA ne s'est pas inversé avec l'ERA. Même si le nombre de syndicats a augmenté et que ce déclin a été enrayé (MAY), le taux de syndicalisation n'a jamais retrouvé son niveau d'avant l'ECA. L'appartenance à un syndicat est restée plus courante dans le secteur public et dans l'industrie manufacturière (MAY, 2004). Dans de nombreuses entreprises et de nombreux secteurs, les gens n'ont jamais réintégré les syndicats. Pour expliquer ce phénomène, MAY (2004) évoque un effet de mimétisme pour toute une génération de travailleurs entrés dans la vie active dans un contexte de vide syndical et de régime de négociations individualisées. Deuxièmement, et ce phénomène est lié au précédent, il a fallu du temps pour parvenir à nouveau à des accords collectifs, négociés par les syndicats, et les négociations individuelles non-syndicales ont encore une place importante dans le système néo-zélandais. Troisièmement, un problème massif de free rider (ou « passager clandestin ») se pose. Comme la couverture des accords collectifs négociés par les syndicats s'étend au-delà des seuls syndiqués, les travailleurs laissent volontiers aux autres le soin d'organiser la lutte sociale et en tirent finalement les éventuels bénéfices. On constate que l'ERA n'a pas véritablement permis de réduire ce phénomène de free riding (WILKINSON, HARBRIDGE, WALSH, 2003).

En 2004, plusieurs amendements à l'ERA ont été adoptés. Pour contrer l'opposition du patronat à la législation de 2000, ils visaient à favoriser la négociation collective et l'adhésion aux syndicats (WILson, 2004). Pour l'essentiel, ils cherchaient à renforcer les pratiques de négociation de bonne foi, 
à limiter le phénomène de free riding en donnant le droit aux syndicats de percevoir une cotisation de négociation auprès des non-syndiqués, à faciliter la signature d'accords collectifs et à consolider les procédures de règlement des griefs. Si le patronat s'est opposé à ces mesures, les syndicats n'y ont vu que des changements marginaux et insuffisants pour réparer les dégâts causés par l'Employment Contracts Act (WILSON, 2004). Outre ces amendements, d'autres textes ont été promulgués pour étendre l'application des dispositions et normes d'hygiène et de sécurité sur le lieu de travail, pour augmenter régulièrement le salaire minimum et pour introduire un congé parental rémunéré et le droit de demander des horaires de travail flexibles (RASMUSSEN, WaLKeR, 2008).

Pour la coalition formée par le parti travailliste et le parti Alliance, il s'agissait de rectifier en douceur les excès engendrés par l'ECA. Le parallèle est possible avec ce qui s'est produit au Royaume-Uni et avec la proposition de l'ALP en Australie en 2009 (négociations de bonne foi, congés parentaux, droit de demander des conditions de travail flexibles). Alors que l'ECA a été radicale dans ses intentions et dans son contenu, la loi ERA constitue, à l'inverse, une tentative modérée de moderniser les conditions de travail et de rétablir les négociations collectives. Les associations patronales se sont opposées à cette législation (malgré la légitimité du mandat gouvernemental) et ont réussi à empêcher l'application effective de certains volets de l'ERA (RASMUSSEN, WALKER, 2008). De leur côté, les syndicats ont estimé que la législation était trop timorée et trop modérée, notamment au regard des chamboulements causés dans le système des relations sociales par l'ECA (WILSON, 2004).

Dans le cas de la Nouvelle-Zélande, la re-régulation partielle des relations sociales n'a pas donné lieu, à court ni à moyen terme, à une recrudescence des adhésions aux syndicats et des négociations collectives. Même si l'objectif de la loi ERA n'était pas de revenir à la situation d'avant la loi ECA (WILSON, 2004), force est de constater que, dans la pratique, l'ECA a provoqué des séquelles tenaces. Dans ce contexte, il n'est pas surprenant que la Nouvelle-Zélande ait fait le choix de procéder à des changements modérés dans les relations sociales, en étendant les droits opposables des travailleurs dans différents domaines (comme les congés parentaux, les temps de travail et les règles d'hygiène et de sécurité sur le lieu de travail) (HowARTH, 2004).
Comparaison du bilan des différents partis travaillistes : en Grande-Bretagne, en Australie et en Nouvelle-Zélande

La Grande-Bretagne, l'Australie et la NouvelleZélande, trois pays ayant cherché à déréguler largement leur marché du travail, ont uniquement réussi à opérer une re-régulation partielle, i.e. à rétablir partiellement le cadre régulateur antérieur à l'ère néolibérale. Dans ces trois cas de figure, les gouvernements travaillistes élus ont annoncé ne pas envisager de revenir vers le passé ; chacun d'eux insistant sur la notion de modernisation et sur l'importance de concevoir des stratégies pour faire face à la mondialisation (WILSON, 2004). Et ce, malgré les liens politiques et financiers traditionnels entre l'aile politique et l'aile syndicale des partis travaillistes et malgré des programmes électoraux remettant en question voire rejetant totalement la législation d'inspiration néolibérale dans le domaine des relations industrielles. Cela ne veut pas dire qu'il n'y a eu aucun changement, au contraire, certains points ont même beaucoup évolué. Au Royaume-Uni, les conditions d'emploi ont été améliorées du fait de l'application des obligations de l'Union européenne en matière sociale, et la Low Pay Commission a obtenu une revalorisation du salaire minimum réel (MetCAlfe, 2008). En Nouvelle-Zélande, les conditions d'emploi se sont améliorées et des mesures ont été prises pour favoriser la progression des négociations collectives. En Australie, les droits et les modalités de protection minimale ont été étendus et les contrats de travail individuels (AWA) abandonnés.

Quels sont donc les obstacles qui empêchent d'abolir totalement l'héritage néolibéral ? Premièrement, des freins institutionnels empêchent une transformation en bloc. En effet, après le déclin des forces syndicales et l'abolition des institutions collectives, il est difficile de réinstaurer un système dont les principales fondations institutionnelles ont été détruites ou très sérieusement abîmées. Cela est particulièrement le cas en Nouvelle-Zélande. Deuxièmement, dans ces trois pays, un fort engagement politique néolibéral persiste dans les rangs même des travaillistes, ce qui confirme ce qu'écrivait MudGe (2008) lorsqu'il remarquait que désormais, le programme néolibéral ne se limitait plus aux seuls partis politiques conservateurs. La Troisième voie britannique, par exemple, a repris à son compte cette notion d'individualisme et prône une large transformation du secteur public. Troisièmement, certaines résistances s'opposent au changement. Dans le cas de la Nouvelle-Zélande, les associations patronales se sont opposées aux mesures, pourtant modérées, introduites par le parti travailliste (RASMUSSEN, Walker, 2008). En Australie, les partis conservateurs ont gardé le contrôle de la Chambre 
haute et pourraient par conséquent chercher à remanier systématiquement les nouvelles mesures visant à re-réguler les relations industrielles.

Alors que la tendance générale est à la re-régulation (limitée) dans ces trois pays, il reste d'importantes disparités dans la forme qu'elle a prise. Comment expliquer à la fois cette similitude et ces différences ? Une explication convaincante de cette coexistence de similitudes et de différences entre la Grande-Bretagne, l'Australie et la Nouvelle-Zélande peut être trouvée dans la nouvelle théorie institutionnaliste, caractérisée, selon WAILES (2000), par la méthodologie de « pression commune et de réponse différenciée ». WAILES et RAMIA (2001, p. 133) citent Locke et THELEN (1995) pour qui, même si la mondialisation a créé des «pressions économiques uniformisées tendant vers une plus grande flexibilité, les types de flexibilité recherchés et les résultats obtenus diffèrent d'un pays à l'autre, en grande partie du fait des particularités des États et des sociétés ». Au cours des années 1980 et 1990 , dans les trois pays couverts par notre analyse, les politiques économiques de dérégulation ont fait écho à des pressions idéologiques et économiques similaires. Même si ces politiques sont toutes d'inspiration néolibérale, leurs conséquences (type et ampleur de la dérégulation) diffèrent en fonction de l'histoire du pays et de l'organisation de ses institutions. Par exemple, le système à chambre unique néo-zélandais - par opposition au régime parlementaire bicaméral australien - a été, selon BRAY et WALSH (1998), un paramètre important ayant facilité la dérégulation radicale du marché du travail en Nouvelle-Zélande en 1991. De même, en Grande-Bretagne, l'absence d'un système d'arbitrage obligatoire qui aurait donné une légitimité institutionnelle aux syndicats a sans doute aidé le gouvernement Thatcher lors de la confrontation avec le syndicat des mineurs en 1984 et 1985.

S'il est généralement admis que les arrangements institutionnels et l'histoire de ces trois pays expliquent en partie la dissemblance des réponses apportées face à des pressions économiques identiques au cours des années 1980 et 1990 , il reste à déterminer quelles ont été les pressions communes qui ont provoqué le phénomène, réel bien que limité, de re-régulation actuel. Une partie de la réponse paraît évidente : les cycles politiques en GrandeBretagne et en Nouvelle-Zélande se sont plus ou moins enchaînés les uns après les autres (l’Australie affichant toujours un certain temps de retard), si bien que les travaillistes, à leur arrivée dans les gouvernements nationaux ont entrepris de re-réguler le marché du travail. Néanmoins, les succès électoraux des travaillistes sont peut-être l'épiphénomène d'une évolution plus fondamentale, née d'un certain rationalisme - en l'occurrence, un certain désaveu des politiques néolibérales et la réticence qui en découle de tolérer davantage de dérégulation. Cela reste une hypothèse théorique, mais certains indices viennent conforter cette idée. En Grande-Bretagne, par exemple, la popularité de certaines œuvres culturelles illustrant les années Thatcher (comme le film Les Virtuoses) et le titre du livre blanc de Tony Blair qui invitait à plus d'Équité au travail reflètent bien le sentiment commun face au néolibéralisme (2). De même, en Australie, la rationalisation des Work Choices, qui ne cadrait pas avec la vie de tous les jours des travailleurs australiens, a été largement perçue comme un acte de bravade de la part d'un gouvernement totalement affranchi de l'opposition du Sénat. En Nouvelle-Zélande, la persistance du manque de vigueur économique en dépit de l'application de politiques néolibérales radicales a détruit toute disposition favorable de l'électorat à poursuivre sur la voie de la dérégulation.

Plusieurs facteurs institutionnels permettent également d'expliquer la nature et l'ampleur de la re-régulation opérée dans chaque pays. Le rôle majeur du mouvement syndical dans le succès du parti travailliste australien en 2007 ne pouvait qu'annoncer une tentative de re-régulation du marché du travail australien par le gouvernement travailliste, et ce, de façon plus vigoureuse qu'en Grande-Bretagne. Néanmoins, même dans ce cas de figure, l'ampleur et la vitesse d'application des changements restent problématiques. La campagne intitulée «Vos droits au travail »[Your Rights@ Work], qui a eu un large poids lors de l'élection de 2007, a toujours un site Internet où les syndicats initiateurs de la campagne continuent d'identifier ce qu'ils estiment être des violations persistantes des droits des salariés et des conditions de travail par la législation Work Choices, toujours en vigueur (Your Rights@Work, 2008). La stratégie du New Labour, qui a fait le choix de se distancier du mouvement travailliste britannique, fait que le mouvement syndical se voit aujourd'hui incapable de jouer un rôle aussi important que par le passé. De même, l'influence d'institutions supranationales comme l'Union européenne a été déterminante dans la forme prise par le phénomène de re-régulation en Grande-Bretagne.

$*$
$* \quad *$

Nous nous sommes attachés à évaluer les difficultés que rencontrent les partis de centre gauche à l'heure de réformer les relations industrielles après le passage de gouvernements conservateurs. Dans les trois pays étudiés dans cet article, les gouvernements conservateurs précédents avaient tenté

(2) Au Royaume-Uni, l'élection récente d'un gouvernement conservateur-libéral démocrate reste à expliquer à l'aune de cette tendance. 
de transformer fondamentalement le système de relations industrielles, notamment en réduisant la présence et l'implication des syndicats et en encourageant les négociations individuelles.

Mais pourquoi est-il si difficile d'infléchir ou de défaire les programmes néolibéraux après un gouvernement conservateur ? Dans ces trois pays, pourtant, le gouvernement nouvellement élu entretient des liens forts avec le mouvement syndical et est animé par la volonté d'améliorer les droits et l'équité au travail. D'après notre analyse, trois raisons pourraient expliquer la persistance des schémas néolibéraux. Premièrement, les différents partis travaillistes ont toujours été partisans de la mondialisation et de la modernisation. Les influences politiques et bureaucratiques du néolibéralisme restent importantes au sein des partis de centre gauche et dans tous les rouages des économies anglo-saxonnes (MudGe, 2008). En Australie, par exemple, nombre de programmes néolibéraux et de politiques de dérégulation majeures ont été appliqués dans le passé par le parti travailliste australien (ALP) lui-même (RAMSAY, BATTIN, 2005). Deuxièmement, la capacité du programme néolibéral à transformer radicalement les relations industrielles ne doit pas être sous-estimée. En Nouvelle-Zélande, par exemple, le tissu syndical a été complètement détruit dans des secteurs entiers, et toute une génération de travailleurs n'a jamais été contact avec un quelconque syndicat (MAY, 2004); difficile dans ces conditions de rétablir quelque chose qui a complètement disparu ! Troisièmement, les différents partis travaillistes se soucient aussi d'entretenir leur légitimité économique auprès de l'électorat et du monde des affaires (RAMSAY, BATTIN, 2005), ce qui implique d'éviter des réformes trop radicales et de favoriser des changements graduels. Enfin, et cela vient s'ajouter aux trois raisons précédentes, il est possible que le changement des structures économiques et sociales ait entraîné, dans chacun de ces trois pays, et indépendamment du parti au pouvoir (parti travailliste ou conservateur), une érosion des fondements des systèmes traditionnels de relations industrielles.

\section{Bibliographie}

Boxall P. (2001), "Evaluating Continuity and Change in the Employment Relations Act 2000", New Zealand Journal of Industrial Relations, Vol. 26, № 1, pp. 27-44.

BrADFord M. (1999), "National Labour Market Policy", New Zealand Journal of Industrial Relations, Vol. 24, № 2, pp. 155-165.

Bray M., Walsh P. (1998), "Different Paths to Neo-Liberalism ? Comparing Australia and New Zealand", Industrial Relations Vol. 37, № 3, pp. 358-87.

Brown W. (2000), "Putting Partnership into Practice in Britain”, British Journal of Industrial Relations, Vol. 38, № 2, pp. 277-299.

Castles F.G., Pierson C. (1996), "A New Convergence? Recent Policy Developments in the United Kingdom, Australia and New Zealand", Policy and Politics, Vol. 24, № 3, pp. 233-246.

DeAKIN S. (1992), "Labour Law and Industrial Relations", in J. Michie (ed.) The Economic Legacy 1979-1992, Academic Press, London.

Dickens L., Hall M. (1995), "The State : Labour Law and Industrial Relations", in P. Edwards (ed.) Industrial Relations : Theory and Practice in Britain, Blackwell, Oxford.

GedDES M. (2005), "Neo-liberalism and Local Governance : Cross national perspectives and speculations", Policy Studies, Vol. 26, № 3-4, pp. 359-377.
George S. (1999), A Short History of Neo-Liberalism, Conference on Economic Sovereignty in a Globalising World, Bangkok, March.

Guest D., Peccei R. (2001), "Partnership at Work : Mutuality and the Balance of Advantage", British Journal of Industrial Relations, Vol. 39 № 2, June, pp. 207-236.

Honeybone A. (1997), "Introducing labour flexibility : The example of New Zealand", International Labour Review, Vol. 134, № 4, pp. 493-507.

Larner W. (2000), "Neo-liberalism : Policy, Ideology, Governmentality", Studies in Political Economy, Vol. 63, 5-25.

MacCallum M. (2007), Poll Dancing: The Story of the 2007 Election, Black Inc, Melbourne.

McKayS. (2001), "Between Flexibility and Regulation : Rights, Equality and Protection at Work", British Journal of Industrial Relations, Vol. 39 № 2 June, pp. 285-303.

MAY R. (2004), "Trade Unions and the Employment Relations Act", in E. Rasmussen (ed.) Employment Relationships, Auckland University Press, Auckland, pp. 159-172.

Metcalf D. (2008), "Why has the British National Minimum Wage had Little or No Impact on Employment ?", Journal of Industrial Relations, Vol. 50, № 3, pp. 489-512.

Mudge S. (2008), "What is Neo-liberalism ?", Socio Economic Review, Vol. 6, № 4, pp. 703-732. 
PeEtz D. (2006), Brave New Work Place, Allen \& Unwin, Sydney.

Prosser, T. (2010), "Public sector cuts loom as coalition government is formed", eironline, http://www.eurofound. europa.eu/eiro/2010/05/articles/uk1005019i.htm, Accessed 16 February, 2011.

RAmsay T., Battin T. (2005), "Labor Party Ideology in the Early 1990s : Working Nation and Paths Not Taken”, Journal of Economic and Social Policy, Vol. 9, No 2, pp. 143-160.

Rasmussen E., Walker B. (2008), Employment Relations in the New Millennium, Department of Employment Relations, Auckland University of Technology.

ShackLeton J. (1998), "Industrial Relations Reform in Britain since 1979", Journal of Labour Research, Vol. 19, No 3, pp. 581-605.

Smith P., Morton, G. (2001), "New Labour's Reform of Britain's Employment Law : The Devil is not only in the Detail but in the Values and Policy Too", British Journal of Industrial Relations, Vol. 39, No 1, pp. 119-138.

Tickell A., Peck, J. (1995), "Self Regulation after Fordism : Regulation Theory, Neo-liberalism and the Global-local Nexus", Economy and Society, Vol. 24, No 3, pp. 357-386.

Trade Union Congress (2011) All Together Campaign, http : //www.tuc.org.uk/theme/index.cfm ? theme=alltogether, Accessed 16 February, 2011.

UNDY R. (1999), "New Labour's 'Industrial Relations Settlement' : The Third Way ?", British Journal of Industrial Relations, Vol. 37, No 2.

Wailes N. (2000), "Economic Change and Domestic Industrial Relations Institutions : Towards a Theoretical
Model", Journal of Industrial Relations, Vol. 24, № 2, pp. 214-233.

Wailes N., Kitay J., Lansbury R. (2008), "Varieties of Capitalism, Corporate Governance and Employment Relations Under Globalisation", in S. Marshall, R. Mitchell and I. Ramsay (eds.) Varieties of Capitalism, Corporate Governance and Employees, Melbourne University Press, Melbourne, pp. 19-38.

Wailes N., Ramia G. (2001), Comparativism and globalisation : An integrated framework for comparing Australian and New Zealand industrial relations, Proceedings of the $15^{\text {th }}$ AIRAANZ Conference, Wollongong, January.

Wilkinson D., Harbridge R., Walsh, P. (2003), "Labour Market Re-regulation and its Effects on Free riding in New Zealand", Journal of Industrial Relations, Vol. 45, No 4, pp. 529-538.

WiLson M. (2004), “The Employment Relations Act : A Framework for a Fairer Way", in E. Rasmussen (ed.) Employment Relationships, Auckland University Press, Auckland.

Wilson R. (2000), “The Decade of Non-compliance : The NZ Government record of Non-compliance with International Labour Standards 1990-98", New Zealand Journal of Industrial Relations, Vol. 25, № 1, pp. 79-94.

Wood S., Goddard J. (1999), “The Statutory Union Recognition Procedure in the Employment Relations Bill : A Comparative Analysis", British Journal of Industrial Relations, Vol. 37, № 2, June, pp. 203-245.

YourRights@Work (2008), http// : www.rightsatwork. com.au/ Accessed 25 August 2009. 